\title{
Revisiting the structure-property relationship of metallic glasses: Common spatial correlation revealed as a hidden rule
}

\author{
Dan Wei, ${ }^{1,2}$ Jie Yang, ${ }^{1,2}$ Min-Qiang Jiang, ${ }^{1,2}$ Bing-Chen Wei, ${ }^{2,3}$ Yun-Jiang Wang, ${ }^{1,2, *}$ and Lan-Hong Dai ${ }^{1,2, \dagger}$ \\ ${ }^{1}$ State Key Laboratory of Nonlinear Mechanics, Institute of Mechanics, Chinese Academy of Sciences, Beijing 100190, China \\ ${ }^{2}$ School of Engineering Science, University of Chinese Academy of Sciences, Beijing 100049, China \\ ${ }^{3}$ Key Laboratory of Microgravity (National Microgravity Laboratory), Institute of Mechanics, Chinese Academy of Sciences, \\ Beijing 100190, China
}

(Received 3 September 2018; revised manuscript received 30 December 2018; published 31 January 2019)

\begin{abstract}
What determines the glass property remains one of the major unsolved problems in both condensed matter physics and materials science. Despite extensive research attempting to identify possible structural features as property signatures in glasses obeying the conventional philosophy of "structure determines property" in matter, the hidden rule about why some proposed structures predict properties effectively but others do not is still poorly understood. Here we revisit some earlier proposed successful "structural descriptors" in glasses, e.g., vibrational mean-squared displacement, flexibility volume, participation fraction of low-frequency vibrational modes, and two-body excess entropy, correlating them with the long-time dynamic property of model glass probed via the activation energy for local structural excitation. We find that all four structural descriptors correlate strongly with the activation energy, presenting large Pearson's correlation coefficients. By examining the spatial nature of the activation energy and the structural descriptors, a common rule for the robustness of structure-property relationships in glasses is established, according to which there exists a critical characteristic correlation length. We further demonstrate the concept that complex structures determine the glass property by manipulating the cutoff distance used to define the two-body excess entropy. Only if this structural descriptor is defined involving atoms beyond the first nearest neighbor does it reproduce the feature of common spatial correlation range in glass. The presence of a common spatial correlation length strongly indicates that it is necessary to include the spatial correlation of a complex structure accounting for the dynamic property of metallic glasses.
\end{abstract}

DOI: 10.1103/PhysRevB.99.014115

\section{INTRODUCTION}

General disordered materials are ubiquitous in nature and have many attractive physical and chemical properties in contrast to their crystalline counterparts. The recent advanced metallic glasses are a category of such amorphous solids with exceptional mechanical functions as promising structural materials, owing to the unique long-range disordered but short-range ordered atomic structure. However, it is difficult to understand their unconventional properties based on the structure, because they do not have distinct lattices and wellrecognized structural defects akin to dislocations in crystals. This challenges the prevailing philosophy of "structure determines property" in materials science and condensed matter physics. The mysterious structure-property relationships constitute one of the most intriguing unsolved problems in the broad community of glassy physics [1-3].

Despite unremitting efforts during the past several decades in recognizing the elementary deformation modes of metallic glasses, it is still unclear how such local structural excitations initiate and where they start. The concepts of free volume [4] and shear transformation [5-7] have been commonly accepted as possible forms of local structural excitations that accommodate the viscoelastic and plastic deformation

\footnotetext{
*yjwang@imech.ac.cn

${ }^{\dagger}$ lhdai@lnm.imech.ac.cn
}

in metallic glasses. However, which kind of microscopic structure facilitates the onset of plasticity in amorphous solids is still puzzling. In other words, identifying the mechanism of irreversible atomic rearrangement under stimuli like temperature and/or external stress $[8,9]$ is the key to understanding the elusive structure-property relationships in disordered solids.

In recent years, several effective "structural descriptors" have been proposed to predict the dynamic atomic arrangement in metallic and colloidal glasses and their supercooled liquids. General structural descriptors as property signatures include local symmetry breaking [10-12], vibrational mean-squared displacement (vibrational MSD) [13-17] and vibrational anisotropy $[18,19]$, localized soft vibrational modes [20-22], flexibility volume [18,19], two-body excess entropy [23], local yielding stress [24] and stress gradient along the deformation path [25], local thermal energy [26], machine-learned structural quantity "softness" [1,27,28], interatomic repulsion softness [29], and a very recent orientational order (Ref. [30]), to name just a few. All these quantities partially predict the nature of localized particle rearrangements or plastic deformation in glasses. However, basic questions arise as to why the conventional straightforward short-range local structures, such as a dislocation's Burger's vector, are not in the aforementioned list of effective structures, and what is the hidden common rule behind these structural descriptors in glasses? 
Recently, a few works have stated that locally preferred geometrical orders, such as local density and simple short-range order, may not be able to predict the dynamic heterogeneity of supercooled liquids and glasses [24], but the spatial correlation between the configurations captures the structural origin of dynamics [1,31-34]. From liquid to glass, a growing static length scale with direct correlation to dynamic heterogeneity is present. The findings also open a window to asserting the structure-property relationships in disordered solids. Perhaps the most straightforward way of examining the utility of a structure is to correlate it with a specific property of glass. Very recently, Cubuk et al. established a solid link between a machine-learned structure "softness" and the particle rearrangement over an extremely wide range of disordered solids with versatile stiffnesses [1]. A hidden governing factor of remarkable commonality in spatial correlation was revealed for the signature of plasticity, which may pave the way to greatly simplifying the understanding of structure-property relationships in glasses.

Molecular dynamics (MD) has proven to be an ideal computational tool to understand the atomic-scale motion mechanisms of glasses and their connection to the structure. However, glasses are in a solid state. The dynamic response of glass to external stimuli usually takes a long time to occur, which far exceeds the classical MD time window, in particular for the temperature range well below the thermal glass transition temperature, i.e., $T_{\mathrm{g}}$ (see Ref. [35]). Therefore, assigning a physically relevant property to the local glass structure is not trivial in amorphous solids. The activation energy extracted from the potential energy landscape (PEL) is recognized as a pivotal physical quantity to characterize the deformation propensity of local structures in amorphous solids $[18,19,36-40]$. It denotes the level of difficulty of an energy state transition from an inherent structure residing in an energy minimum to a neighboring basin on the PEL [41,42]. The activation energy can be estimated via the recently developed activation-relaxation technique nouveau (ARTn) [43-45], which facilitates a quantitative comparison between the structure and property in glasses.

To reveal the hidden rule behind the structure-property relationship of metallic glasses, we systematically investigate the quantitative correlation between four effective structural descriptors and activation energy in a model CuZr glass, as an example of general metallic glasses. It is found that a common spatial correlation range constitutes the most critical factor accommodating the structure-property relationships in metallic glass. The contents of the paper are arranged as follows. After this introduction, we provide details of the simulation procedures and PEL sampling technique in Sec. II. The theoretical background for all four structural descriptors are explained in detail in Sec. III. In Sec. IV, we present the results for the characteristics of PEL and confirm its validity in predicting both athermal and thermal local structural excitations in glass, along with the correlation between structures and property and unveiling the underlying hidden rule. Then, in Sec. V, the critical role of spatial correlation is further discussed in terms of versatilely defined local structural entropies. Finally, we summarize the paper by remarking on the utility of structures in predicting the property in metallic glasses in Sec. VI.

\section{METHODOLOGY}

\section{A. Atomic model of metallic glass}

All atomistic simulations herein are performed using the LAMMPS code [46] on a prototypical binary $\mathrm{Cu}_{50} \mathrm{Zr}_{50}$ model metallic glass as a representative of general amorphous solids. The interatomic interaction is described by a Finnis-Sinclairtype embedded-atom method (EAM) empirical potential [47]. The MD sample contains 19652 atoms, with dimensions of $\sim 70 \times 70 \times 70 \AA^{3}$. The size of the model is far beyond the short-range order $(\sim 3 \AA)$ of the present CuZr glass. The glass sample is prepared by quenching from its equilibrium liquid state at $2000 \mathrm{~K}$ to $0 \mathrm{~K}$, with a high cooling rate of $10^{10} \mathrm{~K} / \mathrm{s}$. An additional sub- $T_{\mathrm{g}}$ annealing at $700 \mathrm{~K}$ is performed for $60 \mathrm{~ns}$ to accelerate the aging dynamics, which drives the inherent structure to the deeper basin on the PEL [48,49]. During quenching, each component of the stress tensor is controlled to be zero within the Parrinello-Rahman barostate [50]. The temperature is controlled by the Nosé-Hoover thermostat [51]. The MD timestep is 2 fs. Periodic boundary conditions (PBCs) are applied on all three directions of the model. After being quenched to $0 \mathrm{~K}$, the sample is additionally in-depth relaxed to a local potential energy minimum by the conjugate gradient (CG) algorithm before studying the structure and property.

\section{B. Sampling activation energy for local structural excitation}

To assign a physically meaningful property to atoms in the glass model, the simulations focus on the single-atom activation energy, which can be regarded as a long-time property effectively characterizing the propensity of the local structural excitation in metallic glasses [52,53]. It is an important physical quantity relevant to the macroscopic mechanical properties and microscopic dynamic heterogeneity of glasses [38-40]. From the perspective of the PEL theory $[41,42]$, the elementary local particle rearrangement process for structural excitation proceeds in two steps. (1) Activation: from an initial local energy minimum on the PEL to a possible connecting saddle state. (2) Relaxation: from the saddle state to a neighboring local energy minimum. The activation energy is the energy difference between the saddle point structure and the structure constrained in the initial local energy minimum. We estimate possible saddle point states of all atoms with single-atom resolution when sampling the local structural excitations via ARTn [43-45]. ARTn is an open-ended software for sampling possible local activation energies in the $3 N$-configurational space of a noncrystalline material starting from an energy basin on the PEL representing an inherent structure of glass.

In the framework of ARTn, the initial perturbation is introduced by imposing a random small displacement on a specific atom or atom cluster in the fully relaxed $0 \mathrm{~K}$ metallic glass model (one inherent structure). In the present work, the activation is restricted to a specific atom by setting the cutoff distance of perturbation to be $2 \AA$, which is shorter than the position of the first peak in the pair correlation function (PDF), $g(r)$, of the glass structure. The magnitude of the initial perturbation displacement is fixed to be $0.1 \AA$, whereas the perturbation direction is chosen randomly for a possible event. The increment movement is set as $0.15 \AA$. When the lowest 
eigenvalue of the Hessian matrix is less than $-0.30 \mathrm{eV} / \AA^{2}$, the system is pushed towards a connecting saddle point along the direction of the lowest normalized eigenvector by using the Lanczos algorithm [54]. Convergence to the saddle point state is attained when the maximum force of all atoms is below $0.05 \mathrm{eV} / \AA$. For statistics purposes, we activate each atom 10 times and finally obtain as many as $\sim 200000$ different activation events accounting for the dynamic heterogeneity of the amorphous model.

\section{THEORY OF STRUCTURAL DESCRIPTORS}

\section{A. Vibrational mean-squared displacement}

The vibrational MSD is written as $\left\langle\Delta r^{2}\right\rangle$. The vibrational MSD of the $i$ th atom is calculated according to the equation

$$
\left\langle\Delta r_{i}^{2}\right\rangle=\left\langle\left|\vec{r}_{i}(t)-\vec{r}_{i, \text { equil }}\right|^{2}\right\rangle_{\tau_{0}}
$$

during a finite time $\tau_{0}$ that includes enough periods of oscillations. $\vec{r}_{i, \text { equil }}$ is the equilibrium position of the $i$ th atom in an inherent structure (after energy minimization by the CG algorithm). Here $\vec{r}_{i}(t)$ is the instantaneous position at different times during tracing for $100 \mathrm{ps}$ at $300 \mathrm{~K}$. Here, the angular brackets imply the time average. Such a suitable time duration guarantees that the atoms vibrate around their thermodynamic equilibrium positions without dynamic hopping between energy basins.

Over the past few decades, the vibrational MSD has proven to be a good indicator of the properties of glassforming liquids, such as the phenomena of slowing down upon cooling [14-17,55] and dynamic heterogeneity [13]. From the perspective of the PEL, the vibrational MSD indicates the thermal average degree of deviation around one local minimum, thereby reflecting the nature of curvature of an energy basin. To be more specific, at a given temperature $T$, it reflects the local configurational constraints considering that the term $k_{\mathrm{B}} T /\left\langle\Delta r^{2}\right\rangle$ is a kind of force constant within the harmonic approximation. Here, $k_{\mathrm{B}}$ is the Boltzmann constant and $k_{\mathrm{B}} T$ is the thermal energy. Thus, the vibrational MSD should be closely related to the rigidity of the solid (elastic modulus) [56], which predicts the energy barrier as rationalized in the cooperative shear model [7]. In this sense, the vibrational MSD can be presented as a straightforward "structural descriptor" of glass.

\section{B. Flexibility volume}

The flexibility volume is a structural parameter proposed by Ding and colleagues $[18,19]$ to quantitatively predict the properties of metallic glass. It has been shown to be strongly correlated with the local shear modulus as well as activation energy for thermal activation in metallic glass [18] and amorphous silicon [19]. The flexibility volume of the $i$ th atom is defined as

$$
v_{\text {flex }, i}=\frac{\left\langle\Delta r_{i}^{2}\right\rangle}{a_{i}^{2}} \Omega_{a, i},
$$

where $\Omega_{a, i}$ is the atomic volume of the $i$ th atom in terms of the 3D Voronoi polyhedron scheme, and $a=\sqrt[3]{\Omega_{a, i}}$ is the average atomic spacing. The flexibility volume is constructed on the vibrational MSD and, therefore, naturally encompasses the thermodynamic information in addition to structure. Combining the configurational and thermodynamic constraints of an atom, it actually represents the free space offered by the local atomic environment in the dynamic response. The flexibility volume efficiently predicts the location of shear transformations in amorphous solids.

\section{Two-body excess entropy}

The two-body excess entropy, or local structural entropy, of the $i$ th atom is defined as

$$
\begin{aligned}
S_{2, i}= & -\frac{1}{2} \sum_{v} \rho_{v} \int_{0}^{r_{\text {cutoff }}}\left\{g_{i}^{\mu v}(\vec{r}) \ln g_{i}^{\mu v}(\vec{r})\right. \\
& \left.-\left[g_{i}^{\mu v}(\vec{r})-1\right]\right\} \mathrm{d} \vec{r},
\end{aligned}
$$

where $v$ and $\mu$ represent the types of particles $(\mathrm{Cu}$ or $\mathrm{Zr}$ atoms in the present case), $\rho_{v}$ is the number density of the $v$-type particles, and $g_{i}^{\mu v}$ denotes the local PDF between the particle $i$ of type $\mu$ and other particles of type $v$. Here $r_{\text {cutoff }}$ is the cutoff distance for the integration. The two-body excess entropy is the two-body term of the excess entropy after an infinite-term expansion $[57,58]$, which represents the loss of configurational entropy over an ideal gas state owing to positional correlation. It has been applied in the prediction of diffusive behavior and heterogeneous dynamics in glassforming liquids [59-62] and in particular to the local particle rearrangement in soft colloidal glasses [23]. Therefore, it could be an intriguing pure structural feature worthy of further investigation in terms of its correlation to the property of glass. Furthermore, the freedom of choice in the cutoff distance provides immense room to examine the critical role of spatial correlation in accommodating the structure-property relationships in amorphous solids.

\section{Participation fraction of low-frequency vibrational modes}

In comparison with most crystals, amorphous solids generally have a large excess of low-frequency vibrational modes, which are found to be spatially quasilocalized [21,32,6370] and regarded as the "soft spots" in glassy materials [22,71-73]. Such low-frequency vibrational modes are claimed to be strongly correlated with shear transformations in metallic glass [22] and causally correlated to the irreversible structural reorganization of the particles in supercooled liquids [20]. The soft modes are therefore recommended here as one of the effective "structural descriptors" to predict the properties of glasses.

The participation fraction of the low-frequency vibrational modes of a particle $i$ is given by [20,22]

$$
p_{i}=\sum_{\omega}\left|\vec{e}_{\omega}^{i}\right|^{2}
$$

where $\vec{e}_{\omega}^{i}$ is the corresponding polarization vector of particle $i$ in the normal mode characterized by the vibrational frequency $\omega$. The normal mode analysis is performed by diagonalization of the dynamic matrix of the inherent structure of glass after energy minimization with the CG algorithm [20,22]. The force constants are derived based on the EAM potential. Here, the summation $\sum_{\omega}$ is performed over the $0.25 \%$ lowestfrequency normal modes for the $i$ th particle as its participation fraction. 

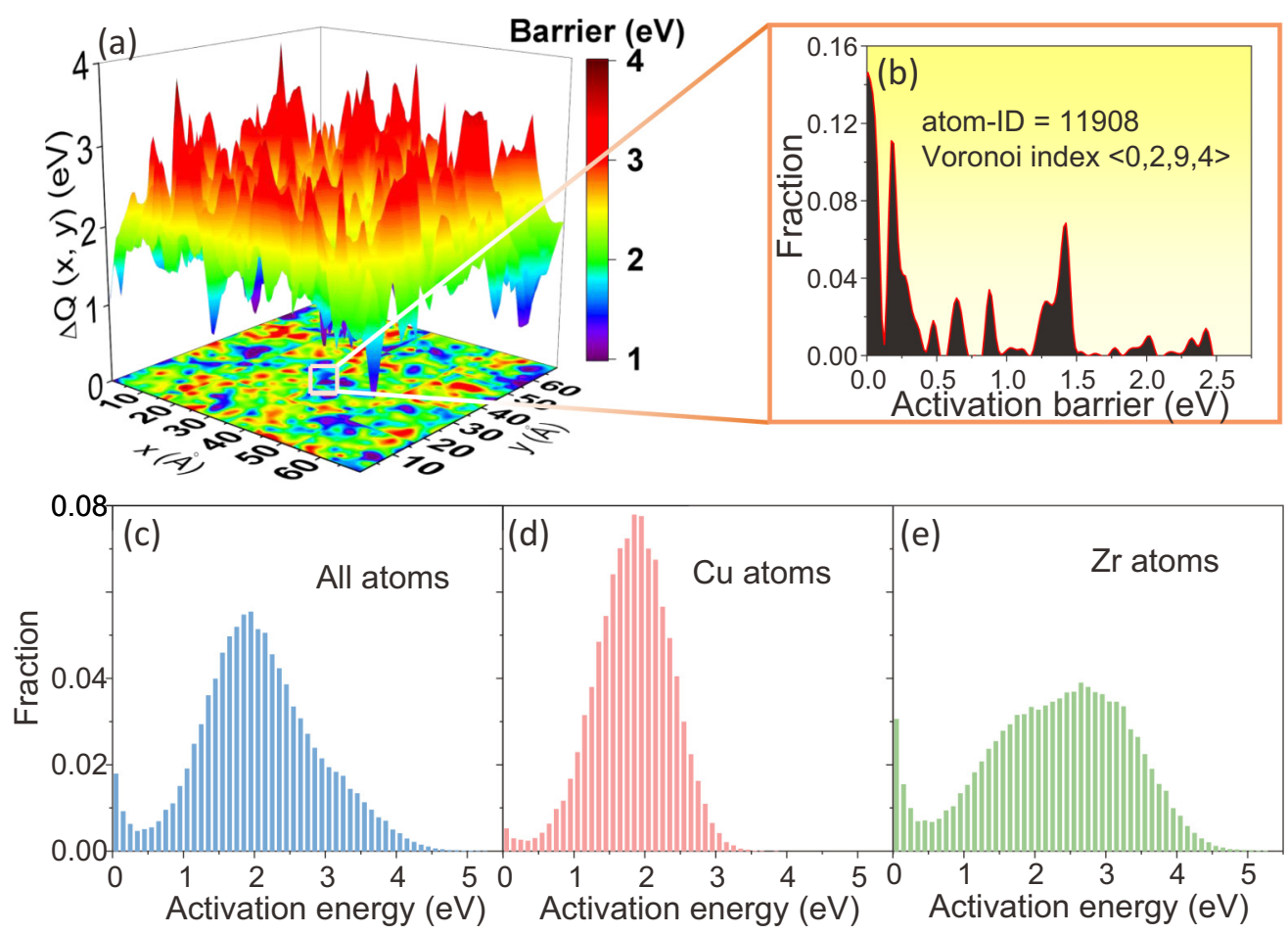

FIG. 1. Features of the PEL in $\mathrm{Cu}_{50} \mathrm{Zr}_{50}$ metallic glass. (a) Three-dimensional color map of the spatial distribution of local activation energies. The local barriers are spatially correlated. The low or high barriers are prone to stay together. The shown slice is normal to the $z$ direction with a thickness of $3.9 \AA$ A. (b) Activation energy spectrum of a specific Zr atom (ID 11908), with the local structure in Voronoi index $\langle 0,2,9,4\rangle$, after being activated 1000 times along random directions. The wide distribution of barriers indicates the complexity of the PEL in amorphous solids. (c)-(e) Histograms of activation energies for all atoms, $\mathrm{Cu}$ atoms, and $\mathrm{Zr}$ atoms, respectively.

\section{RESULTS}

\section{A. Features of the potential energy landscape}

As metallic glass is a promising structural material, in this section we focus on its mechanical properties. Shear transformation has been widely regarded as the basic deformation mechanism of metallic glass [5,6]; it is a thermally activated local structural excitation with the help of temperature and/or mechanical work. The activation of such a particle rearrangement process corresponds to the energy state transformation between neighboring basins (inherent structures) on the PEL. Starting from an inherent structure located in a local energy minimum on the PEL, there are many possible different minimum energy pathways linking to neighboring local basins. When being stimulated by temperature or stress, the system will escape from the sub-basin to a neighboring basin along the corresponding minimum-energy pathway with a saddle point separating the two local energy minima. The energy difference between the saddle point structure and the structure of the initial energy minimum is defined as the activation energy, or energy barrier $\Delta Q$, of a local structural excitation. It is a very critical physical parameter quantifying the incubation timescale of the activation of a plastic event. Therefore, it can be regarded as a long-time property of glasses. We sample the distribution of activation energies using the ARTn method. In Fig. 1, the features of the PEL and the statistics of the activation energies of the studied $\mathrm{Cu}_{50} \mathrm{Zr}_{50}$ glass are summarized.

First, the PEL of general amorphous solids is much more complicated than that of their crystalline counterparts owing to the lack of periodicity in structure. In Fig. 1(a), we show the spatial distribution of activation energies in a slice chosen on the $x-y$ plane of the model. The thickness of the slice is $3.9 \AA$ along the $z$ direction, which corresponds to the first trough of the PDF (short-range order). The 3D plot of the topological information on activation energy is extremely rugged, indicating the complexity of the PEL in glass. For example, here we choose a specific Zr atom with atom ID 11908 in the system for demonstration. Although the local short-range structure of the atom is unique in terms of the Voronoi index of $\langle 0,2,9,4\rangle$, its dynamic property is extremely scattered. The Voronoi index $\left\langle n_{3}, n_{4}, n_{5}, n_{6}\right\rangle$ is a vector of integers constituting a histogram of the number of edges on the faces of the 3D Voronoi cell, and $n_{i}$ is the number of $i$-edged faces [74]. In Fig. 1(b), we show the histogram of activation energies for this $\mathrm{Zr}$ atom after activating 1000 times with a random initial perturbation direction. Multiple peaks can be clearly observed in the wide-distributed spectrum. In the $3 N$-configurational space, there is immense room for activations starting from one local region. The wide distribution of barriers for a specific atom is strong evidence of the lack of a one-to-one intuitive structure-property correlation in the obscure glassy structure, at least in the sense of short-range structure. This observation is in agreement with earlier findings that the local density or short-range order possesses very weak correlation to atom rearrangement in a model glass [24]. Instead, statistics structures and activation energies should be considered when discussing the structure-property relationships in noncrystalline materials, owing to the inherent structural and dynamic 
heterogeneity. Finally, we note from the projection of the peaks shown in Fig. 1(a) (bottom plane) that atoms possessing lower (blue) or higher (red) activation energies tend to stay together, which indicates that the long-time property of glass is spatially correlated.

The whole spectra of the activation energies for the $\mathrm{Cu}_{50} \mathrm{Zr}_{50}$ system, $\mathrm{Cu}$ atoms, and $\mathrm{Zr}$ atoms are shown in Figs. 1(c)-1(e), respectively. The activation energies are distributed over a very broad range, spreading from $\sim 0$ to $5 \mathrm{eV}$, which signifies the versatility of dynamic structures in the glassy state. All spectra can be decomposed into two distribution modes, namely an exponential decaying mode at low activation energies and a Rayleigh mode in the medium to high activation energy regime. The former is akin to the feature of liquid dynamics with a cascade process, whereas the latter corresponds to solidlike structures in which the activations behave like a localized process [38-40]. The left tail of the activation energy spectrum usually dominates the dynamics of glass, because such events take place within a short time under specific conditions of temperature and stress. Our samplings are consistent with the results of previous PEL samplings on a similar $\mathrm{Cu}_{56} \mathrm{Zr}_{44}$ metallic glass [38-40]. One may also note that the distribution of activation energies for $\mathrm{Cu}$ atoms is more concentrated than that of $\mathrm{Zr}$ atoms. This is because $\mathrm{Zr}$ atoms have a higher coordination number, which yields greater diversity in the local structural environment. The peak position of the $\mathrm{Zr}$ barrier distribution shifts to the right compared with that of $\mathrm{Cu}$ atoms. This is because $\mathrm{Zr}$ atoms are heavier than $\mathrm{Cu}$ atoms, and the former are hard to activate under the same conditions. In other words, $\mathrm{Zr}$ atoms experience larger constraints in $\mathrm{CuZr}$ glass than $\mathrm{Cu}$ atoms. Finally, there is a greater fraction of $\mathrm{Zr}$ atoms obeying the exponential decay mode according to the histograms shown in Fig. 1(e). This phenomenon possibly originates from the slower dynamics of $\mathrm{Zr}$ atoms (compared with $\mathrm{Cu}$ atoms) during the preparation of the sample by the standard heatingquenching technique in $\mathrm{MD}$. Compared with $\mathrm{Cu}$ atoms, the $\mathrm{Zr}$ atoms take longer time to relax into stable positions corresponding to deeper basins on the PEL.

\section{B. Validity of activation energy in predicting local structural excitation}

\section{Prediction of athermal excitation}

To confirm that the activation energy is a pivotal physical quantity in measuring the dynamic properties of glasses, we consider two special cases with external stimulus of either pure mechanical stress or pure temperature. The former stimulus is provided by following the protocol of athermal quasistatic shear (AQS) deformation [75], and the latter stimulus is provided by tracing the mobility of atoms at a finite temperature. We investigate the validity of the activation energy in predicting the two dynamic properties of glasses.

In Fig. 2, we demonstrate the robustness of the activation energy in predicting the athermal local structure excitation driven only by the stress in glasses. Figures 2(a)-2(d) show the first four rearrangement events corresponding to the stress drops on the stress-strain curve of AQS loading, as indicated in the four insets in the panels. The background contours illustrate the levels of activation energies on a slice with thickness of $3.9 \AA$ in the undeformed model. The activation energies are therefore coarse grained on such a thickness normal to the shown plane. The location and size of the white bubbles superimposed on the contours denote the spatial information and magnitude of the athermal events. The size of the bubble is proportional to the level of atomic strain calculated based on the configurations just before and after the stress drop. The atomic strain is quantified by the local minimum nonaffine squared displacement, $D_{\min }^{2}$, proposed by Falk and Langer [6],

$$
\begin{aligned}
D_{\min }^{2}= & \frac{1}{N_{\mathrm{c}}} \sum_{j}\left\{\vec{r}_{j}(t)-\vec{r}_{i}(t)\right. \\
& \left.-\mathbf{J}_{i}\left[\vec{r}_{j}(t-\Delta t)-\vec{r}_{i}(t-\Delta t)\right]\right\}^{2} .
\end{aligned}
$$

where the atom $i$ is surrounded by $j \in N_{\mathrm{c}}$ atoms within a reasonable cutoff distance, $\vec{r}$ is the position vector of an atom, and $\mathbf{J}_{i}$ is the local deformation gradient tensor that best minimizes the affine deformation component. Here, $t$ and $\Delta t$ denote the time and time interval between the instantaneous and reference configurations, respectively. As expected, the spatially isolated events with atoms experiencing major rearrangement are located precisely in the low-activation-energy regions (blue regimes in the contour plot). Note that the location of activation is also dependent on the shear protocol, e.g., $x y$ simple shear in the present case. As a result, not all the low-barrier regions can be activated simultaneously. In $\mathrm{AQS}$, priority is given to the most stress-sensitive event in a specific loading form. Nonetheless, the activation energy can precisely predict the occurrence of athermal activation. It should be regarded as a quite reasonable physical parameter for predicting the deformation of glasses.

\section{Prediction of thermal excitation}

In the case of thermal activation, we test two cases that are relevant to the short-time and long-time properties of glasses in terms of vibrational MSD and diffusive MSD. The results are explained in Figs. 3(a) and 3(b), respectively. Again, the contours show a spatial distribution of activation energy on a chosen slice of the inherent structure. On one hand, one can see the fairly good correspondence between vibrational MSD and activation energy in Fig. 3(a). Here the sizes of bubbles denote the magnitude of vibrational MSD by considering the fluctuation amplitude of atoms at $300 \mathrm{~K}$ for $100 \mathrm{ps}$. For clarity, we only show the deviation of vibrational MSD to the ensemble average value, i.e., $\sqrt{\mathrm{vMSD}}-\overline{\sqrt{\mathrm{vMSD}}}$, to reduce the thermal noise. A scaling factor of 60 is assigned for better visualization of vibrational MSD. The vibrational MSD is actually a mechanical feature closely related to the force constant (elasticity) at thermodynamic equilibrium. It reflects the configurational constraint owing to the atomic environment. Therefore, we confirm that the atomic-scale activation energy is a good dynamic signature of the short-time thermodynamic property. This is easy to understand because the activation energy is positively proportional to the local shear modulus, as proposed by Argon in his shear transformation model [5] and Johnson and Samwer in their cooperative shear model [7]. 

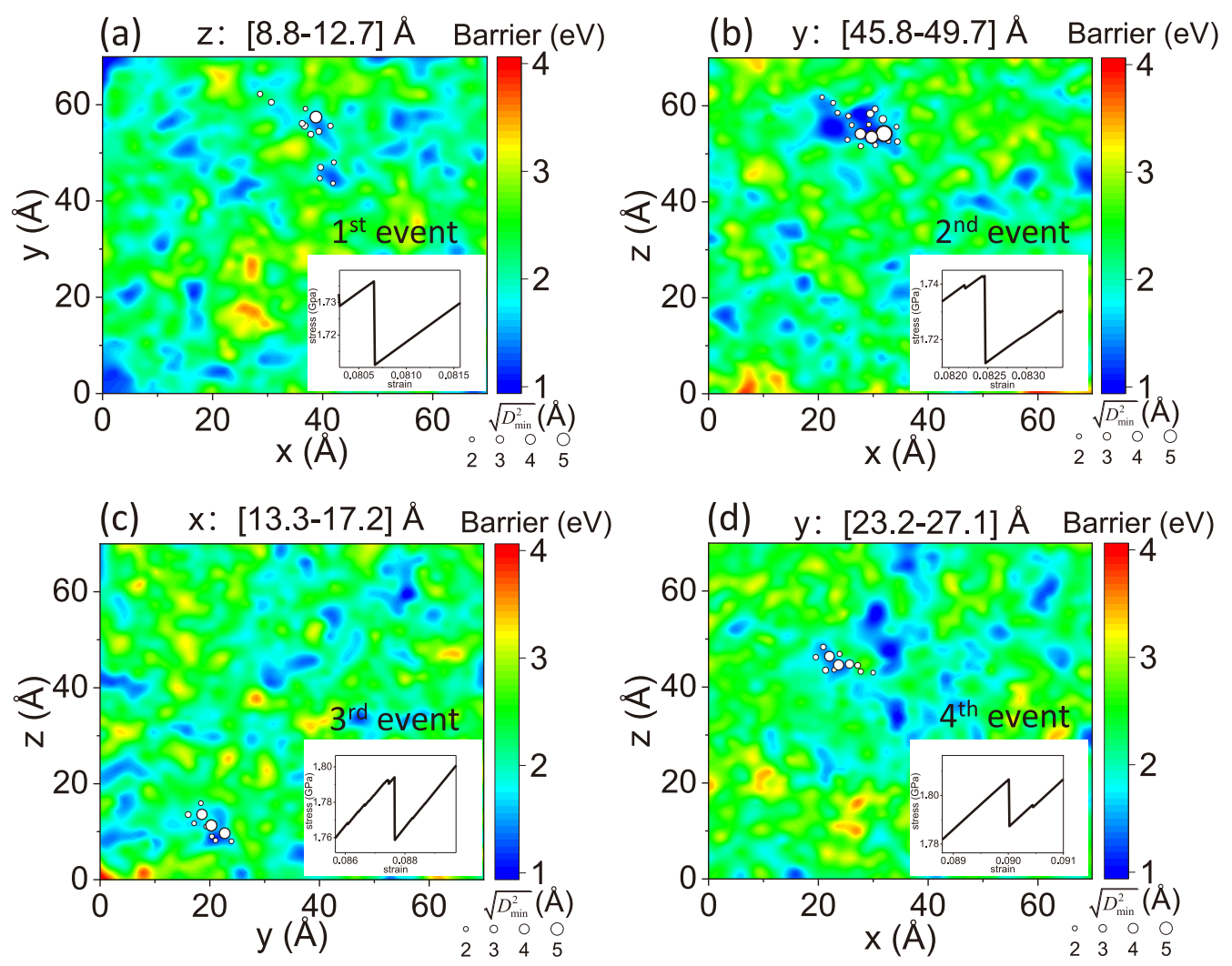

FIG. 2. Prediction of athermal local structural excitation via the activation energy. (a)-(d) First four athermal events during AQS deformation and the corresponding activation energy contour maps before deformation. The contour maps denote the activation energy field in the slices where atomic rearrangements occur. The slices are $3.9 \AA$ thick. The location and dimension of the athermal events are indicated by the white bubbles. The size of the bubbles denotes the magnitude of nonaffine displacement in terms of $D_{\min }^{2}$. The four insets in the panels are the instantaneous fragments of the AQS stress-strain curve, in which the stress drops indicate the occurrence of athermal events.

On the other hand, we also investigate the correlation between the activation energy and diffusive MSD, which represents real thermally activated hopping between basins on the PEL. The results are shown in Fig. 3(b). As explained in the log plot of the diffusive MSD-time relationship in the inset of Fig. 3(b), we determine the magnitude of the real diffusive MSD at $700 \mathrm{~K}$ after a $50 \mathrm{~ns}$ MD run. This time duration is already far beyond the diffusion plateau that brings about real escape from the energy minimum. The size of the white bubbles denotes the magnitude of deviation to the ensemble average diffusive MSD, e.g., $\sqrt{\mathrm{MSD}}-\overline{\sqrt{\mathrm{MSD}}}$ with a scaling factor of 1.5. It is noted that although atom hopping basically occurs in the low-barrier regions, the one-to-one correspondence is poor as compared with vibrational MSD, as shown in Fig. 3(a). This is expected because the thermal activation is a statistic result, especially at high temperature. If enough samplings are performed, one can obtain a better correlation between the barrier and mobility. Another reason is that the long-time MD run at $700 \mathrm{~K}$ may lead to extra relaxation, which could change the original inherent structure, yielding relatively less correlation between the mobility of the atoms and the original activation energy field. However, the typical trend is that low-activation-energy atoms have a greater propensity to move. The activation energy can also be regarded as a long-time property signature of glassy structures.

\section{Quantitative structure-property relationships}

The belief "structure determines property" is the foundation of a vast majority of the studies in materials science and condensed matter physics. The well-known line defectdislocation -in crystals presents the most successful example of this philosophy in the deformation and mechanical properties of crystalline materials. However, in the case of noncrystalline solids, e.g., metallic glass, there is no simple structural descriptor that can be regarded as the "defect" of the materials that dominates the materials' functions. Despite the difficulty, in the past decades, there have still been a few successful general "structural descriptors" of glasses that can predict the shear transformation or local atomic rearrangement in glasses and their supercooled liquid counterparts. A basic question arises as to what is the hidden rule behind the effective structural descriptors in property prediction, whereas other simple short-range structures cannot establish a one-to-one correlation with property. For example, one unique Voronoi short-range structure may show a very wide range of properties in terms of activation energy, as demonstrated in Fig. 1(b).

To answer this question, we revisit the correlation between the activation energy and four effective structural descriptors, i.e., (1) vibrational MSD $\left\langle\Delta r_{i}^{2}\right\rangle$, (2) flexibility volume $v_{\text {flex }, i}$, (3) two-body excess entropy $S_{2, i}$, and (4) participation fraction of low-frequency vibrational modes $p_{i}$, to check whether they are correlated and, if so, identify the physical mechanism 

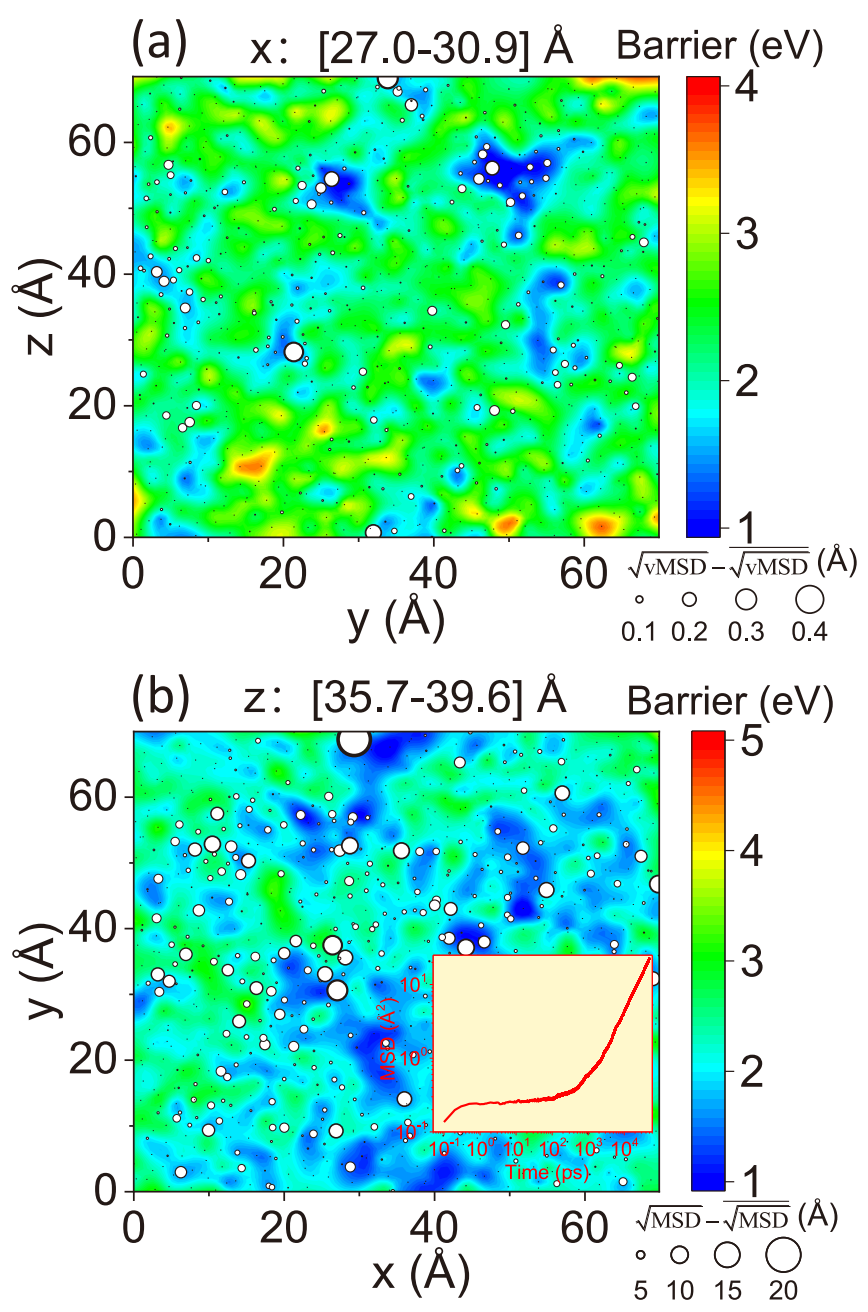

FIG. 3. Prediction of thermally activated diffusion via activation energy. Correlation between activation energy and (a) vibrational MSD, as well as (b) diffusive MSD. The contour maps show the spatial distribution of activation energy within the slices normal to the $x$ and $z$ directions, respectively, with a thickness of $3.9 \AA$. The bubbles superimposed on the contour maps indicate the positions of the atoms experiencing pronounced vibrational or diffusive MSD. The size of bubbles denotes the magnitude of deviation to the ensemble average values of the vibrational MSD, $\sqrt{\text { vMSD }}-\overline{\sqrt{\text { vMSD }}}$ and diffusive MSD, $\sqrt{\mathrm{MSD}}-\overline{\sqrt{\mathrm{MSD}}}$ in (a) and (b), respectively. For clarity, a scaling factor of 60 is assigned for vibrational MSD and 1.5 for diffusive MSD.

underlying the structure-property relationships of glasses. The results are summarized in Fig. 4 by plotting the atomicresolution activation energy against the aforementioned four structures. Each data point denotes a coarse graining of $\sim 200$ atoms sorted by the magnitude of quantity describing the structural descriptor. One can see from the scattered data of Fig. 4 that there exists a strong correlation between the activation energy and structure descriptors, either for $\mathrm{Cu}$ or $\mathrm{Zr}$ atoms. Thus, these four proposed structures can be regarded as the anticipated structural signatures of properties in glasses.

Furthermore, we show the best linear regression fit on all the scattered data shown in Fig. 4, which yields the Pearson's correlation coefficient $r_{\mathrm{P}}$ that quantifies the robustness of the proposed structure-property correlations in glasses. The absolute values of all derived $\left|r_{\mathrm{P}}\right|$ are listed in Fig. 5 for $\mathrm{Cu}$ and $\mathrm{Zr}$ atoms. Most correlation coefficients are larger than 0.95 , which indicates robust structure-property relationships except in the case of the $\Delta Q-S_{2, i}$ correlation for $\mathrm{Zr}$ atoms. This is possibly because $\mathrm{Zr}$ atoms tend to be at higher positions on the PEL that are less stable, compared with $\mathrm{Cu}$ atoms during cooling in the glass preparation process. However, the four structures are already very satisfactory predictors of the glass property. The common feature underlying these structural descriptors and the activation energy must play an important role in the structure-property relationships in glasses, which is discussed in detail later in this paper.

Whereas the correlation between the structure and property is pronounced for all the surveyed physical quantities as shown in Fig. 4, the physical background underlying the correlations is not necessarily the same. First, the vibrational MSD is a thermodynamic feature that describes the curvature of the energy basin on the PEL. In particular, the ratio between the thermal energy and vibrational amplitude, $k_{\mathrm{B}} T /\left\langle\Delta r_{i}^{2}\right\rangle$, denotes the force constant of the atom in the framework of harmonic approximation. A higher vibrational MSD implies lower stiffness and smaller curvature; hence, atoms experience less constraints and can undergo real hopping more easily. The correlation also indicates that the height of the energy peak (saddle point) can usually be informed by the narrowness of the trough (local minimum) on the PEL. In the language of materials science, the local shear modulus is proportional to the activation energy of shear transformation in glass [5,7].

The flexibility volume is constructed on the vibrational MSD [18]. In addition to atomic rigidity, it also includes the short-range volumetric constraint within a Voronoi cell. It is therefore regarded as a physically structural quantity combining both thermodynamic and configurational information. The strong correlation between the activation energy and flexibility volume is not surprising, because vibrational MSD is already a good signature of property, whereas the Voronoi volume of the atoms is not very scattered.

The two-body excess entropy $S_{2}$ of local structural reflects the loss of configurational entropy over their ideal gas state owing to positional correlation. It can be also regarded as a local structural entropy of an atom. The atom with higher $S_{2}$ is less correlated with the atomic environment. In other words, they are more disordered and for more microscopic states in the local structure. Such a group of atoms with more structural diversity is nearer to the ideal gas state that is highly rejuvenated compared with the ordered atoms and is ready to flow like fluid. Therefore, atoms with high two-body excess entropy are easy to move and have lower activation energy.

Atoms with a high participation fraction of low-frequency vibrational modes have been proposed as the "soft spot" in glass and supercooled liquids, which can be regarded as an effective structural predictor of irreversible particlelevel rearrangement in amorphous materials [21,22]. The low-frequency modes are usually localized in space, which is closely related to the well-known Boson peak (excess vibrational modes compared with the Debye squared model) in the generally disordered materials. It has been shown that the low-frequency vibrational anomaly is a possible origin of primary $\alpha$ and secondary $\beta$ relaxation, rationalized by an 
(a)

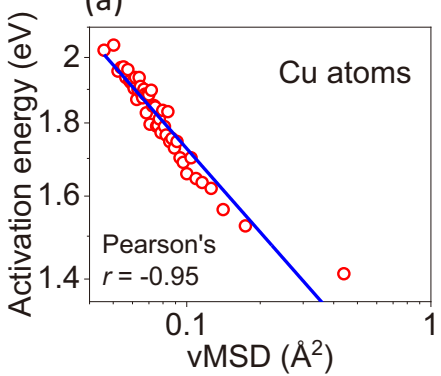

(e)

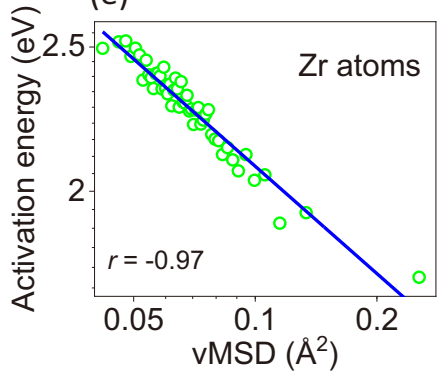

(b)

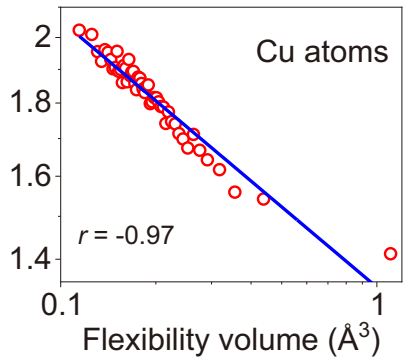

(f)

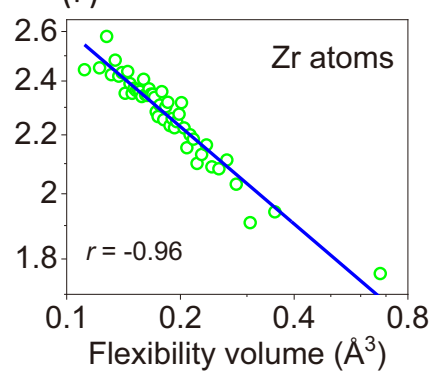

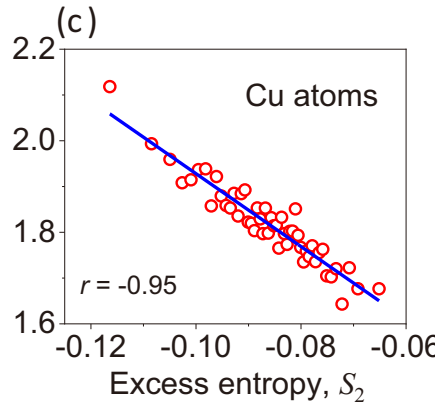

(g)

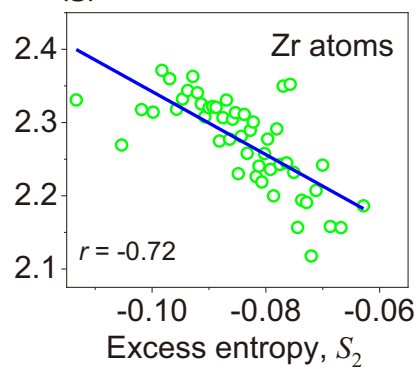

(d)

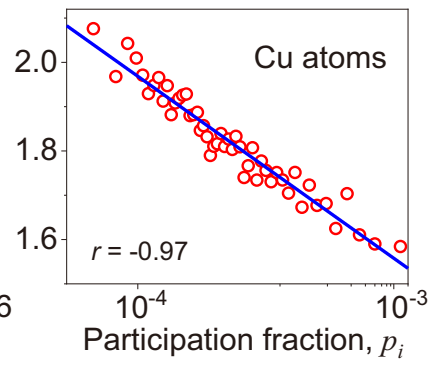

(h)

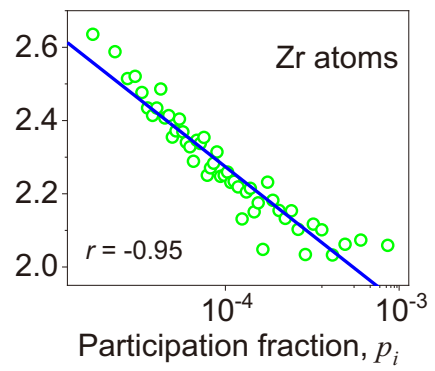

FIG. 4. Quantitative correlation between activation energy and the four effective structural descriptors. The surveyed structural descriptors include: (1) vibrational MSD $\left\langle\Delta r_{i}^{2}\right\rangle$, (2) flexibility volume $v_{\text {flex }, i}$, (3) two-body excess entropy $S_{2, i}$, and (4) participation fraction of lowfrequency vibrational modes $p_{i}$. Activation energies of $\mathrm{Cu}$ and $\mathrm{Zr}$ atoms are sorted based on the magnitude of the quantity describing each structural descriptor with a bin size of $\sim 200$ atoms. The correlations are shown in (a)-(d) for Cu atoms and in (e)-(h) for $\mathrm{Zr}$ atoms. The best-fit linear regression lines are shown in each panel together with the Pearson's correlation coefficients $r_{\mathrm{P}}$.

atomic-scale viscoelastic theory of metallic glass [76,77]. It is therefore the participation fraction $p_{i}$ that can quantitatively predict the activation energy of local structural excitation.

\section{Hidden rule underlying the robustness of structure- property relationships}

After confirming the validity of the four structural descriptors in predicting the property of glass, a basic question arises as to why the above-mentioned structural descriptors can predict the dynamic property quantitatively whereas a unique short-range Voronoi structure presents a wide range of activation energies, as shown in Fig. 1(b). To identify the hidden rule underlying the mysterious structure-property correlation in glass, we further show the spatial characteristics of the activation energy and the four structural descriptors

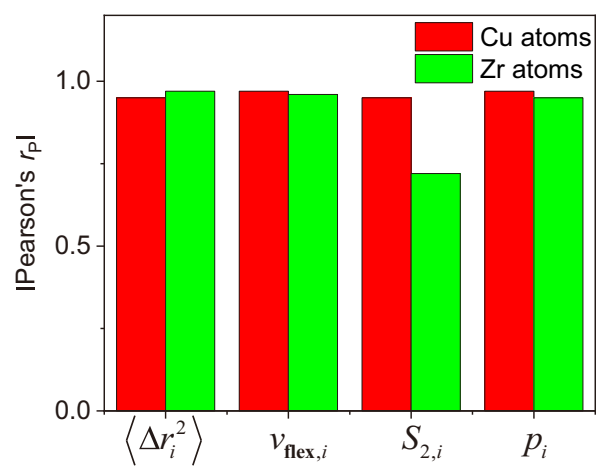

FIG. 5. Absolute values of Pearson's correlation coefficient $\left|r_{\mathrm{P}}\right|$ between the activation energy and four structural descriptors, derived from the plots shown in Fig. 4. in Fig. 6. Figures 6(a)-6(e) show the contour maps of the activation energy and the quantities describing the nature of structural descriptors, on the same slice normal to the $z$ direction in the model. It is seen that all the spatial patterns of the structural descriptors are self-similar, and they also resemble the spatial feature of the activation energy. The size of the spatial heterogeneity is of the order of a nanometer. The common spatial nature of the effective structures and property indicates the important role of spatial correlation in determining the mechanical property of glassy materials.

To quantify the common spatial nature, we calculate the spatial autocorrelation functions of the four structural descriptors and activation energy according to

$$
C(r)=\frac{\left\langle\Delta P_{r_{0}} \Delta P_{r_{0}+r}\right\rangle-\left\langle\Delta P_{r_{0}}\right\rangle^{2}}{\left\langle\Delta P_{r_{0}}^{2}\right\rangle-\left\langle\Delta P_{r_{0}}\right\rangle^{2}},
$$

where $P$ denotes a physical property, namely, the above structural descriptors and activation energy. Here $\Delta P_{r_{0}}=P_{r_{0}}-\bar{P}$ is the deviation to the ensemble average property $\bar{P}$ for the $i$ th atom at a reference position $r_{0}$, and $\Delta P_{r_{0}+r}$ is the same deviation of the atoms locating at a distance of $r$ from the reference position. Here, the angle brackets $\langle\cdots\rangle$ denote the ensemble average. The calculated spatial autocorrelation functions are shown in Figs. 6(f)-6(j) as a function of the distance. On the one hand, they are almost identical. Evidently, all the correlation functions decrease exponentially with respect to distance $r$. Our observation is in agreement with previous findings on the size of shear transformation zones [78] and the correlation length of atomic flexibility volume in amorphous silicon [19]. On the other hand, the correlation functions are always positive, which implies that atoms with similar structure and activation energy have greater propensity to stay together spatially. 


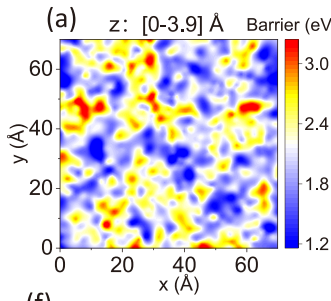

$$
\text { (f) }
$$
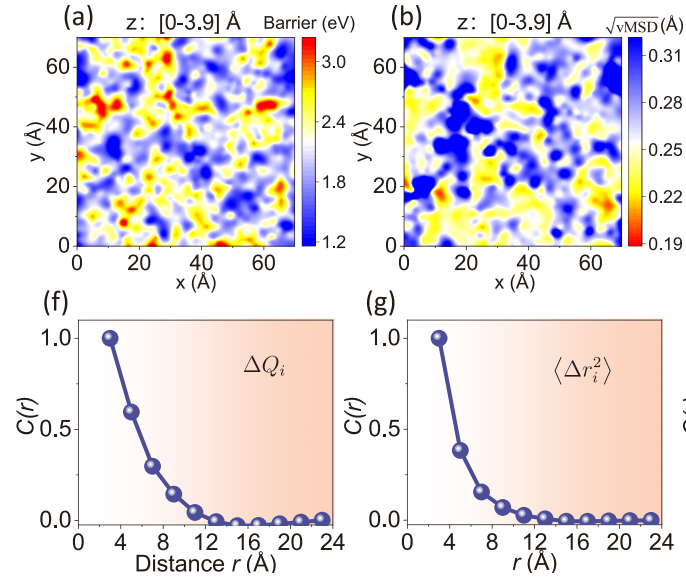

(g)

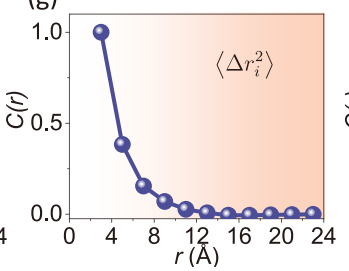

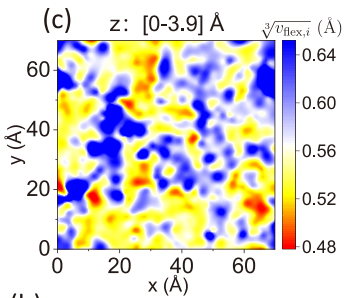

(h)

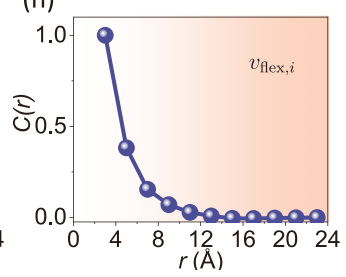

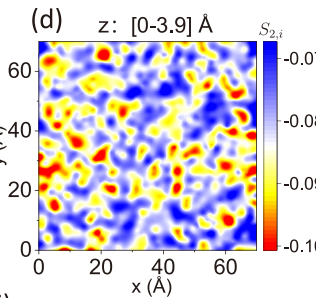

(i)

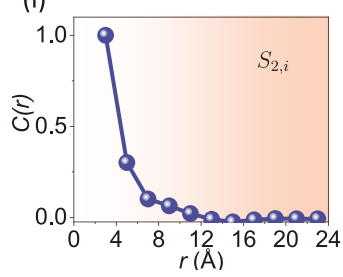

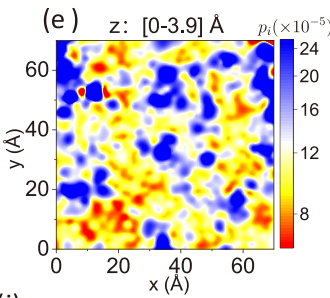

(j)

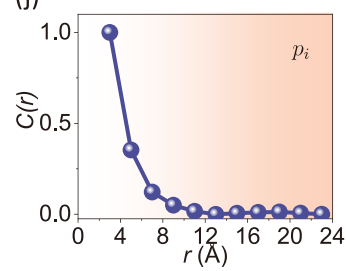

FIG. 6. Spatial correlation behind the structure-property relationship. (a)-(e) Spatial distributions of the activation energy and the four surveyed structural descriptors. From left to right: activation energy $\Delta Q_{i}$, vibrational MSD $\left\langle\Delta r_{i}^{2}\right\rangle$, flexibility volume $v_{\text {flex }, i}$, two-body excess entropy $S_{2, i}$, and participation fraction of low-frequency vibrational modes $p_{i}$, respectively. The shown slice is normal to the $z$ direction with a thickness of $3.9 \AA$ A. The self-similarity of the contour maps indicate the validity of structural descriptors in predicting properties, which also implies possible common nature underlying the structures and property. (f)-(j) Spatial autocorrelation functions of the activation energy and the four structural descriptors. The first value of the correlation function is set to be unity.

To further understand the spatial correlation of the glass structure and property, we fit all the correlation functions according to an empirical equation $C(r) \propto \exp (-r / \xi)$ as used in the literature [1], where $\xi$ is defined as the correlation length, which represents the smallest distance beyond which the correlation function is less than $\exp (-1) \approx 0.37$. The fits of the correlation functions are shown in Fig. 7 in a semilogarithmic manner. By definition, the correlation function decays to 0.37 at the correlation length, i.e., $r=\xi$. However, the correlation functions have been normalized to unity at a distance of $3 \AA$ for convenience. As a result, the fitted functions have been shifted to the right by $3 \AA$. Therefore, the true correlation length should be $3 \AA$ shorter than the

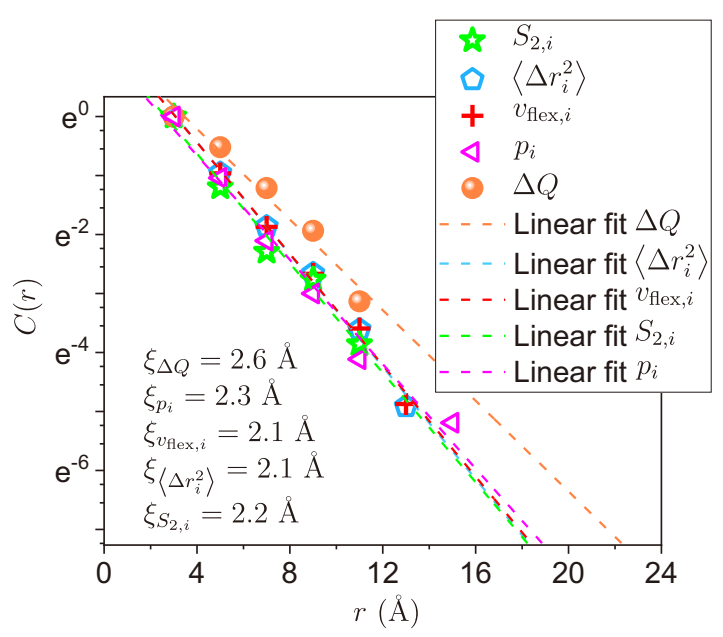

FIG. 7. Semilogarithmic plot of the spatial autocorrelation functions versus distance for activation energy and the four structural descriptors. The dashed lines are the best empirical fits to $C(r) \propto$ $\exp (-r / \xi)$, which defines the correlation length, $\xi$. The correlation lengths for all the structural descriptors are $\sim 2.2 \AA$, which is close to that of $\xi=2.6 \AA$ for a property (i.e., activation energy). apparent length at which $C(r)=\exp (-1)$ as shown in Fig. 7 . However, the rigid shift of correlation function in the distance does not affect the determination of $\xi$, because only the slopes of semilogarithmic plots matter in Fig. 7.

It is seen from Fig. 7 that the spatial autocorrelation lengths of all four structural descriptors are approximately $2.2 \AA$, which are similar to that of the activation energy ( $2.6 \AA)$. They are all close to the average nearest neighbor distance, i.e., $r_{\mathrm{NN}}=2.8 \AA$, determined from the position of the first peak in the PDF. It suggests an intimate relationship between the structural features and dynamic property of metallic glass via a common spatial correlation length scale. For comparison, we also calculate the spatial autocorrelation function of a simple short-range order parameter, i.e., the local fivefold symmetry (L5FS) parameter used to identify the local Voronoi structure [10], as shown in Fig. S1 of the Supplemental Material (SM) [79]. Figures S1(a) and S1(b) denote that there does not exist strong correlation between L5FS and activation energy for either $\mathrm{Cu}$ or $\mathrm{Zr}$ atoms. Figure $\mathrm{S} 1$ (c) shows that the spatial autocorrelation of L5FS decreases exponentially with respect to distance. Its quantified autocorrelation length in Fig. S1(d) is only $1.2 \AA$, much shorter than that of the activation energy. Hence, the spatial correlation of the structure could be a hidden rule underlying the structure-property relationship of metallic glasses. From another perspective, the spatial correlation could mean the sweeping scale of the "defectlike" structure or dynamic property. The premise behind the effective prediction for dynamic property is that the sweeping scale of the "defectlike" is consistent with that of dynamic excitation. The insensitive prediction from the simple SRO like L5FS mainly originates from the complexity of amorphous solids: Only one simple static parameter cannot describe the atomic environment well; even the same SRO parameter can lead to very different properties, as shown in Fig. 1(b). Of course, if one extends the simple SRO to the complex structure defined involving atoms at longer distance (e.g., interconnection of icosahedral cluster $[80,81]$ ), the 
prediction can be improved. Both of those two factors need to be considered if one intends to search for an effective structural descriptor of the general amorphous solids. In other words, a simple short-range structure does not work in predicting the property, whereas a complex super structure defined by multiple atoms in the cluster up to the common correlation length may be responsible for the property of glasses. Our observation of the spatial correlation between structure and property is in agreement with earlier studies on different noncrystalline systems [1,82]. The spatial correlation length of the machine-learned structure "softness" and particle rearrangement are of the order of the particle size in Ref. [1]. As has been demonstrated by Wang et al., the spatial correlation of elastic heterogeneity can tune macroscopic deformation modes of metallic glasses [82]. Finally, we note that the spatial correlation length is defined as the distance where the correlation function decays to $\exp (-3) \approx 0.05$ in the literature [82], but the point is that how to choose the method of definition does not affect the relative magnitudes of the spatial correlation length scale between structure and property.

\section{DISCUSSION}

We now further discuss the critical role of the spatial correlation in determining the property of glasses, in terms of the two-body excess entropy. Returning to its definition shown in Eq. (3), there exists a critical cutoff distance, $r_{\text {cutoff }}$, which gives a converged value of a single atom $S_{2, i}$. This cutoff distance of the integral has been chosen reasonably as the fourth trough $(11.2 \AA)$ of $g(r)$ in calculating $S_{2, i}$ because the fluctuation of $g(r)$ almost vanishes at this distance; see Fig. 8(a). Beyond this distance, the atoms' surroundings make no contribution to $S_{2, i}$.

On the other hand, manipulating the cutoff distance $r_{\text {cutoff }}$ would provide further insights into the question why the simple short-range order structure cannot be a good property predictor in glass. The freedom in cutoff distance provides immense room to demonstrate the critical role of the spatial correlation length in determining the property of glasses. To this end, we redefine the two-body excess entropy at different cutoff distances, i.e., 3.9, 6.4, 8.8, and $11.2 \AA$, which correspond to the positions of the first, second, third, and fourth troughs of $g(r)$, respectively. In this way, the quantity can be transformed from a simple short-range order parameter involving only the first nearest neighbor atoms to a complex structure defined with atoms at longer distance without changing anything else, as we shorten the cutoff distance of the integration in Eq. (3). The spatial autocorrelation functions of these newly defined two-body excess entropies are displayed in Fig. 8(b). They have similar features with positive values and decay with distance. However, the correlation functions decay increasingly quickly with decreasing cutoff distance, which implies that the spatial correlation length $\xi$ becomes increasingly shorter as $S_{2, i}$ varies from complex structure to simple short-range order. In other words, the structure defined at the nearest-neighbor distance does not suffice to define a successful property descriptor in glasses.
The spatial autocorrelation functions of $S_{2, i}$ shown in Fig. 8(b) are further displayed in Fig. 8(c) in a semilogarithmic manner. In this way, we can quantify the correlation length by fitting the data empirically to $C(r) \propto \exp (-r / \xi)$. The derived correlation length is shown in Fig. 8(d) as a function of the cutoff distance. As one can see, $\xi$ increases with $r_{\text {cutoff }}$ initially, whereas it saturates at a longer distance of $11.2 \AA$, which corresponds to the fourth shell of the positional correlation of atoms. By demonstrating the cutoffdistance-modulated spatial correlation of the two-body excess entropy, we may have answered the question "why should the structure-property correlation be established on complex structure" in glasses. If only one structural descriptor were composed of atoms within a reasonable spatial range (not only the atoms within the first nearest neighbor), it would be effective in correlating the property of glasses. Otherwise, the simple short-range structure, which does not contain complete physical information and necessary atoms in its definition up to the common spatial correlation length, would not define an effective complex structure as a property descriptor of glasses, in analogy to the role that dislocation plays in the deformation of crystals. Finally, we stress that possessing the common spatial correlation length for one structural feature is fundamental to predict the dynamic feature of glass. That is, the spatial correlation is intrinsically embedded in the relationship between the structure and property of disordered materials [1]. This means that any structural parameter that does not possess the common correlation length scale would not be a good property predictor. In a broad sense, the spatial correlation can be a reasonable reference for testing the utility of one structural feature in determining the property of glass.

\section{CONCLUDING REMARKS}

In summary, we have systematically revisited the structure-property relationships in a model $\mathrm{CuZr}$ metallic glass by examining the quantitative correlation between four structural features and the dynamic property via atomicresolution activation energy. The operation is aimed at answering a general question regarding the kind of structural feature that would be meaningful in determining the function of a glass. The four structural descriptors at the atomic scale include the vibrational MSD, flexibility volume, two-body excess entropy, and participation fraction of low-frequency vibrational modes. All four structure descriptors have been proposed in the literature as effective structural signatures of the dynamic property of glasses and supercooled liquids. The dynamic property of glass here is represented by the activation energy of a local structural excitation sampled on the $3 N$-configurational PEL, which is confirmed as quite a physically meaningful quantity in characterizing either athermal or thermal deformation of metallic glass. Whereas a unique simple short-range Voronoi cluster presents a wide spectrum of activation energies, all four surveyed structural descriptors are correlated strongly with the activation energy, quantified with a significant fraction of Pearson's correlation coefficient. After checking the spatial feature of the structure field and activation energy field, we unveil a common critical correlation length as the physical factor underlying 
(a)

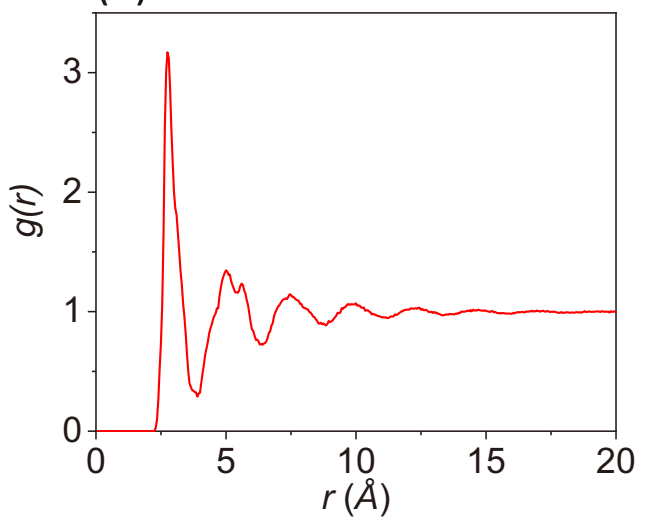

(c)

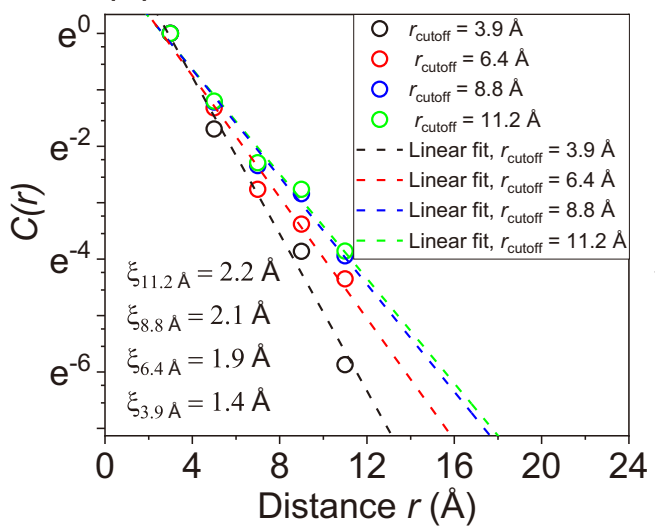

(b)

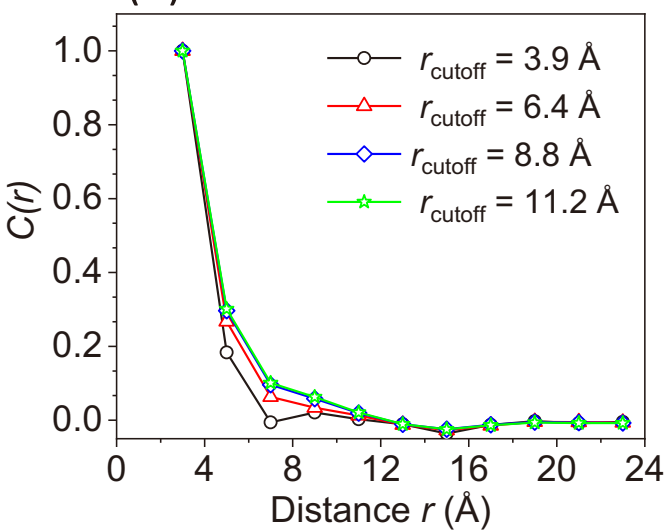

(d)

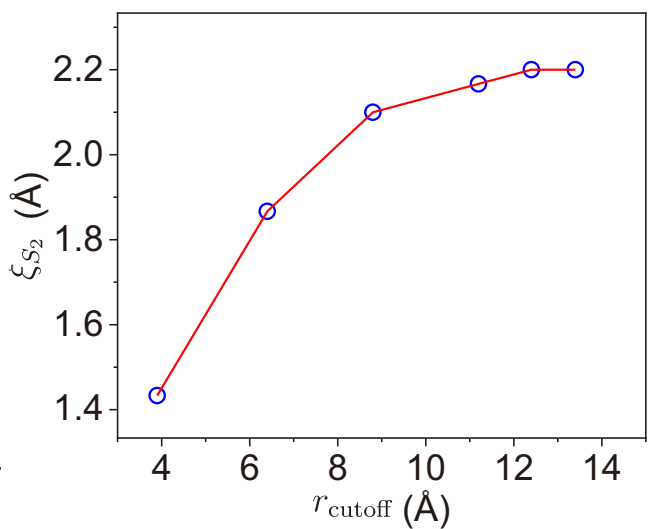

FIG. 8. Demonstration of the critical role of the spatial correlation underlying the robustness of the structure-property relationship of glasses. (a) Pair correlation function $g(r)$ of the model glass. (b) Spatial autocorrelation functions for two-body excess entropy defined with various cutoff distances, i.e., 3.9, 6.4, 8.8, and $11.2 \AA$, respectively. They correspond to the first, second, third, and fourth troughs of $g(r)$, respectively. (c) Semilogarithmic plot of the autocorrelation functions in (b). The dashed lines are empirical fits to $C(r) \propto \exp (-r / \xi)$. (d) Correlation length $\xi$ increases with the magnitude of the cutoff distance $r_{\text {cutoff }}$, saturating at the fourth trough of $g(r)$, i.e., $r_{\text {cutoff }}=11.2 \AA$.

the robustness of structure-property relationships in glasses. The concept of common spatial correlation as the hidden rule of an effective property descriptor has been further discussed by manipulating the cutoff distance in defining one of the structural descriptors, i.e., the two-body excess entropy. Should local structural entropy predict the property of atoms, it has to be defined beyond the fourth shell of $g(r)$ for glass to include necessary physical information and atoms. The common spatial correlation length actually means the accordant scale and scope for the "defectlike" structure and dynamic excitation. It could be as a bond linking structure and property.

Hence, we might have an ad hoc answer for the philosophical question of whether structure determines property in glass. On one hand, no. Simple short-range structures such as the Voronoi polyhedron and the coordination number cannot well predict the dynamic property of glasses. On the other hand, yes. A complex structure descriptor defined by multiple atoms within a cluster up to several shells of its surroundings can predict the dynamic feature accurately. The hidden rule behind the robustness of the structure-property relationship in metallic glasses is the spatial correlation of a specific structural descriptor. Our findings provide comprehensive insights into the mysterious structure-property relationships in metallic glasses. We have explained the physical reason for which the complex structure can more effectively predict the dynamic property of glass. Moreover, the common correlation length scale can serve as a general guide to identify more physically elegant and more efficient property signatures of disordered materials.

\section{ACKNOWLEDGMENTS}

We thank Jeppe Dyre, Peter Harrowell, and Daniel Gianola for very insightful discussions. We acknowledge financial support from the National Key Research and Development Program of China (No. 2017YFB0702003 and No. 2017YFB0701502), NSFC (No. 11672299 and No. 11790292), the Strategic Priority Research Program (No. XDB22040303), the Key Research Program of Frontier Sciences (No. QYZDJSSW-JSC011), and the Youth Innovation Promotion Association of CAS (No. 2017025). The calculations were performed on the "Era" petascale supercomputer of the Computer Network Information Center of CAS. 
[1] E. D. Cubuk, R. J. S. Ivancic, S. S. Schoenholz, D. J. Strickland, A. Basu, Z. S. Davidson, J. Fontaine, J. L. Hor, Y.-R. Huang, Y. Jiang, N. C. Keim, K. D. Koshigan, J. A. Lefever, T. Liu, X.-G. Ma, D. J. Magagnosc, E. Morrow, C. P. Ortiz, J. M. Rieser, A. Shavit, T. Still, Y. Xu, Y. Zhang, K. N. Nordstrom, P. E. Arratia, R. W. Carpick, D. J. Durian, Z. Fakhraai, D. J. Jerolmack, D. Lee, J. Li, R. Riggleman, K. T. Turner, A. G. Yodh, D. S. Gianola, and A. J. Liu, Structure-property relationships from universal signatures of plasticity in disordered solids, Science 358, 1033 (2017).

[2] C. A. Schuh, T. C. Hufnagel, and U. Ramamurty, Mechanical behavior of amorphous alloys, Acta Mater. 55, 4067 (2007).

[3] T. C. Hufnagel, C. A. Schuh, and M. L. Falk, Deformation of metallic glasses: Recent developments in theory, simulations, and experiments, Acta Mater. 109, 375 (2016).

[4] F. Spaepen, A microscopic mechanism for steady state inhomogeneous flow in metallic glasses, Acta Metall. 25, 407 (1977).

[5] A. S. Argon, Plastic deformation in metallic glasses, Acta Metall. 27, 47 (1979).

[6] M. L. Falk and J. S. Langer, Dynamics of viscoplastic deformation in amorphous solids, Phys. Rev. E 57, 7192 (1998).

[7] W. L. Johnson and K. Samwer, A Universal Criterion for Plastic Yielding of Metallic Glasses with a $\left(T / T_{g}\right)^{2 / 3}$ Temperature Dependence, Phys. Rev. Lett. 95, 195501 (2005).

[8] P. Guan, M. W. Chen, and T. Egami, Stress-Temperature Scaling for Steady-State Flow in Metallic Glasses, Phys. Rev. Lett. 104, 205701 (2010).

[9] Y.-J. Wang, M. Zhang, L. Liu, S. Ogata, and L. H. Dai, Universal enthalpy-entropy compensation rule for the deformation of metallic glasses, Phys. Rev. B 92, 174118 (2015).

[10] H. L. Peng, M. Z. Li, and W. H. Wang, Structural Signature of Plastic Deformation in Metallic Glasses, Phys. Rev. Lett. 106, 135503 (2011).

[11] Z. W. Wu, M. Z. Li, W. H. Wang, and K. X. Liu, Hidden topological order and its correlation with glass-forming ability in metallic glasses.Nat. Commun. 6, 6035 (2015).

[12] R. Milkus and A. Zaccone, Inversion-symmetry breaking controls the boson peak anomaly in glasses and crystals, Phys. Rev. B 93, 094204 (2016).

[13] A. Widmer-Cooper, P. Harrowell, and H. Fynewever, How Reproducible are Dynamic Heterogeneities in a Supercooled Liquid, Phys. Rev. Lett. 93, 135701 (2004).

[14] J. C. Dyre, Colloquium: The glass transition and elastic models of glass-forming liquids, Rev. Mod. Phys. 78, 953 (2006).

[15] L. Larini, A. Ottochian, C. De Michele, and D. Leporini, Universal scaling between structural relaxation and vibrational dynamics inglass-forming liquids and polymers, Nat. Phys. 4, 42 (2008).

[16] J. C. Dyre and W. H. Wang, The instantaneous shear modulus in the shoving model, J. Chem. Phys. 136, 224108 (2012).

[17] B. A. Pazmiño Betancourt, P. Z. Hanakata, F. W. Starr, and J. F. Douglas, Quantitative relations between cooperative motion, emergent elasticity, and free volume in model glassforming polymer materials, Proc. Natl. Acad. Sci. U.S.A. 112, 2966 (2015).

[18] J. Ding, Y. Q. Cheng, H. Sheng, M. Asta, R. O. Ritchie, and E. Ma, Universal structural parameter to quantitatively predict metallic glass properties, Nat. Commun. 7, 13733 (2016).
[19] Z. Fan, J. Ding, Q. J. Li, and E. Ma, Correlating the properties of amorphous silicon with its flexibility volume, Phys. Rev. B 95, 144211 (2017).

[20] A. Widmer-Cooper, H. Perry, P. Harrowell, and D. R. Reichman, Irreversible reorganization in a supercooled liquid originates from localized soft modes, Nat. Phys. 4, 711 (2008).

[21] A. Widmer-Cooper, H. Perry, P. Harrowell, and D. R. Reichman, Localized soft modes and the supercooled liquid's irreversible passage through its configuration space, J. Chem. Phys. 131, 194508 (2009).

[22] J. Ding, S. Patinet, M. L. Falk, Y. Cheng, and E. Ma, Soft spots and their structural signature in a metallic glass, Proc. Natl. Acad. Sci. U.S.A. 111, 14052 (2014).

[23] X. Yang, R. Liu, M. Yang, W. H. Wang, and K. Chen, Structures of Local Rearrangements in Soft Colloidal Glasses, Phys. Rev. Lett. 116, 238003 (2016).

[24] S. Patinet, D. Vandembroucq, and M. L. Falk, Connecting Local Yield Stresses with Plastic Activity in Amorphous Solids, Phys. Rev. Lett. 117, 045501 (2016).

[25] B. Xu, M. L. Falk, J. F. Li, and L. T. Kong, Predicting Shear Transformation Events in Metallic Glasses, Phys. Rev. Lett. 120, 125503 (2018).

[26] J. Zylberg, E. Lerner, Y. Bar-sinai, and E. Bouchbinder, Local thermal energy as a structural indicator in glasses, Proc. Natl. Acad. Sci. U.S.A. 114, 7289 (2017).

[27] E. D. Cubuk, S. S. Schoenholz, J. M. Rieser, B. D. Malone, J. Rottler, D. J. Durian, E. Kaxiras, and A. J. Liu, Identifying Structural Flow Defects in Disordered Solids Using MachineLearning Methods, Phys. Rev. Lett. 114, 108001 (2015).

[28] S. S. Schoenholz, E. D. Cubuk, D. M. Sussman, E. Kaxiras, and A. J. Liu, A structural approach to relaxation in glassy liquids, Nat. Phys. 12, 469 (2016).

[29] J. Krausser, K. H. Samwer, and A. Zaccone, Interatomic repulsion softness directly controls the fragility of supercooled metallic melts, Proc. Natl. Acad. Sci. U.S.A. 112, 13762 (2015).

[30] J. Yang, Y.-J. Wang, E. Ma, A. Zaccone, L. H. Dai, and M. Q. Jiang, Structural Parameter of Orientational Order to Predict the Boson Vibrational Anomaly in Glasses, Phys. Rev. Lett. 122, 015501 (2019).

[31] J. Russo and H. Tanaka, Assessing the role of static length scales behind glassy dynamics in polydisperse hard disks, Proc. Natl. Acad. Sci. U. S. A. 112, 6920 (2015).

[32] S. Gelin, H. Tanaka, and A. Lemaître, Anomalous phonon scattering and elastic correlations in amorphous solids, Nat. Mater. 15, 1177 (2016).

[33] H. Tong and H. Tanaka, Revealing Hidden Structural Order Controlling Both Fast and Slow Glassy Dynamics in Supercooled Liquids, Phys. Rev. X 8, 011041 (2018).

[34] Y.-C. Hu, Y.-W. Li, Y. Yang, P.-F. Guan, H.-Y. Bai, and W.H. Wang, Configuration correlation governs slow dynamics of supercooled metallic liquids, Proc. Natl. Acad. Sci. U.S.A. 115, 6375 (2018).

[35] D. Rodney, A. Tanguy, and D. Vandembroucq, Modeling the mechanics of amorphous solids at different length scale and time scale, Modell. Simul. Mater. Sci. Eng. 19, 083001 (2011).

[36] D. Rodney and C. Schuh, Distribution of Thermally Activated Plastic Events in a Flowing Glass, Phys. Rev. Lett. 102, 235503 (2009).

[37] D. Rodney and C. A. Schuh, Yield stress in metallic glasses: The jamming-unjamming transition studied through Monte 
Carlo simulations based on the activation-relaxation technique, Phys. Rev. B 80, 184203 (2009).

[38] Y. Fan, T. Iwashita, and T. Egami, How thermally activated deformation starts in metallic glass, Nat. Commun. 5, 5083 (2014).

[39] Y. Fan, T. Iwashita, and T. Egami, Crossover from Localized to Cascade Relaxations in Metallic Glasses, Phys. Rev. Lett. 115, 045501 (2015).

[40] Y. Fan, T. Iwashita, and T. Egami, Energy landscape-driven nonequilibrium evolution of inherent structure in disordered material, Nat. Commun. 8, 15417 (2017).

[41] F. H. Stillinger, A topographic view of supercooled liquids and glass formation, Science 267, 1935 (1995).

[42] P. G. Debenedetti and F. H. Stillinger, Supercooled liquids and the glass transition, Nature (London) 410, 259 (2001).

[43] G. T. Barkema and N. Mousseau, Event-Based Relaxation of Continuous Disordered Systems, Phys. Rev. Lett. 77, 4358 (1996).

[44] R. Malek and N. Mousseau, Dynamics of lennard-jones clusters: A characterization of the activation-relaxation technique, Phys. Rev. E 62, 7723 (2000).

[45] E. MacHado-Charry, L. K. Béland, D. Caliste, L. Genovese, T. Deutsch, N. Mousseau, and P. Pochet, Optimized energy landscape exploration using the ab initio based activation-relaxation technique, J. Chem. Phys. 135, 034102 (2011).

[46] S. Plimpton, Fast parallel algorithms for short-range molecular dynamics, J. Comput. Phys. 117, 1 (1995).

[47] M. I. Mendelev, M. J. Kramer, R. T. Ott, and D. J. Sordelet, Molecular dynamics simulation of diffusion in supercooled $\mathrm{Cu}-$ Zr alloys, Philos. Mag. 89, 109 (2009).

[48] F. Zhang, M. I. Mendelev, Y. Zhang, C. Z. Wang, M. J. Kramer, and K. M. Ho, Effects of sub- $T_{g}$ annealing on $\mathrm{Cu}_{64.5} \mathrm{Zr}_{35.5}$ glasses: A molecular dynamics study, Appl. Phys. Lett. 104, 061905 (2014).

[49] Y. Zhang, C. Z. Wang, M. I. Mendelev, F. Zhang, M. J. Kramer, and K. M. Ho, Diffusion in a $\mathrm{Cu}-\mathrm{Zr}$ metallic glass studied by microsecond-scale molecular dynamics simulations, Phys. Rev. B 91, 180201(R) (2015).

[50] M. Parrinello and A. Rahman, Polymorphic transitions in single crystals: A new molecular dynamics method, J. Appl. Phys. 52, 7182 (1981).

[51] S. Nosé, A molecular dynamics method for simulations in the canonical ensemble, Mol. Phys. 52, 255 (1984).

[52] S. Swayamjyoti, J. F. Löffler, and P. M. Derlet, Local structural excitations in model glasses, Phys. Rev. B 89, 224201 (2014).

[53] S. Swayamjyoti, J. F. Löffler, and P. M. Derlet, Local structural excitations in model glass systems under applied load, Phys. Rev. B 93, 144202 (2016).

[54] E. Cancès, F. Legoll, M.-C. Marinica, K. Minoukadeh, and F. Willaime, Some improvements of the activation-relaxation technique method for finding transition pathways on potential energy surfaces, J. Chem. Phys. 130, 114711 (2009).

[55] U. Buchenau and R. Zorn, A relation between fast and slow motions in glassy and liquid selenium, Europhys. Lett. 18, 523 (1992).

[56] S. Saw and P. Harrowell, Rigidity in Condensed Matter and Its Origin in Configurational Constraint, Phys. Rev. Lett. 116, 137801 (2016).

[57] D. C. Wallace, On the role of density fluctuations in the entropy of a fluid, J. Chem. Phys. 87, 2282 (1987).
[58] A. Baranyai and D. J. Evans, Direct entropy calculation from computer simulation of liquids, Phys. Rev. A 40, 3817 (1989).

[59] T. S. Ingebrigtsen and H. Tanaka, Structural predictor for nonlinear sheared dynamics in simple glass-forming liquids, Proc. Natl. Acad. Sci. U.S.A. 115, 87 (2017).

[60] M. Dzugutov, A universal scaling law for atomic diffusion in condensed matter, Nature (London) 381, 137 (1996).

[61] A. Samanta, Sk. M. Ali, and S. K. Ghosh, New Universal Scaling Laws of Diffusion and Kolmogorov-Sinai Entropy in Simple Liquids, Phys. Rev. Lett. 92, 145901 (2004).

[62] H. Tanaka, T. Kawasaki, H. Shintani, and K. Watanabe, Criticallike behavior of glass-forming liquids, Nat. Mater. 9, 324 (2010).

[63] L. E. Silbert, A. J. Liu, and S. R. Nagel, Vibrations and Diverging Length Scales Near the Unjamming Transition, Phys. Rev. Lett. 95, 098301 (2005).

[64] A. Rahman, M. J. Mandell, and J. P. McTague, Molecular dynamics study of an amorphous Lennard -Jones system at low temperature, J. Chem. Phys. 64, 1564 (1976).

[65] M. Wyart, S. R. Nagel, and T. A. Witten, Geometric origin of excess low-frequency vibrational modes in weakly connected amorphous solids, Europhys. Lett. 72, 486 (2005).

[66] A. Ghosh, V. Chikkadi, P. Schall, and D. Bonn, Connecting Structural Relaxation with the Low Frequency Modes in a HardSphere Colloidal Glass, Phys. Rev. Lett. 107, 188303 (2011).

[67] A. J. Liu and S. R. Nagel, Granular and jammed materials, Soft Matter 6, 2869 (2010).

[68] V. Mazzacurati, G. Ruocco, and M. Sampoli, Low-frequency atomic motion in a model glass, Europhys. Lett. 34, 681 (1996).

[69] D. Kaya, N. Green, C. E. Maloney, and M. F. Islam, Normal modes and density of states of disordered colloidal solids, Science 329, 656 (2010).

[70] P. Tan, N. Xu, A. B. Schofield, and L. Xu, Understanding the Low-Frequency Quasilocalized Modes in Disordered Colloidal Systems, Phys. Rev. Lett. 108, 095501 (2012).

[71] N. Xu, V. Vitelli, A. J. Liu, and S. R. Nagel, Anharmonic and quasilocalized vibrations in jammed solids - modes for mechanical failure, Europhys. Lett. 90, 56001 (2010).

[72] M. L. Manning and A. J. Liu, Vibrational Modes Identify Soft Spots in a Sheared Disordered Packing, Phys. Rev. Lett. 107, $108302(2011)$

[73] A. Tanguy, B. Mantisi, and M. Tsamados, Vibrational modes as a predictor for plasticity in a model glass, Europhys. Lett. 90, 16004 (2010).

[74] H. W. Sheng, W. K. Luo, F. M. Alamgir, J. M. Bai, and E. Ma, Atomic packing and short-to-medium-range order in metallic glasses, Nature (London) 439, 419 (2006).

[75] C. E. Maloney and A. Lemaître, Amorphous systems in athermal, quasistatic shear, Phys. Rev. E 74, 016118 (2006).

[76] B. Cui, J. Yang, J. Qiao, M. Jiang, L. Dai, Y.-J. Wang, and A. Zaccone, Atomic theory of viscoelastic response and memory effects in metallic glasses, Phys. Rev. B 96, 094203 (2017).

[77] B. Cui, J. F. Gebbia, J.-L. Tamarit, and A. Zaccone, Disentangling $\alpha$ and $\beta$ relaxation in orientationally disordered crystals with theory and experiments, Phys. Rev. E 97, 053001 (2018).

[78] M. Zink, K. Samwer, W. L. Johnson, and S. G. Mayr, Plastic deformation of metallic glasses: Size of shear transformation zones from molecular dynamics simulations, Phys. Rev. B 73, 172203 (2006). 
[79] See Supplemental Material at http://link.aps.org/supplemental/ 10.1103/PhysRevB.99.014115 for demonstrating the spatial autocorrelation of an extra structural descriptor defined with shortrange order.

[80] J. Ding, Y.-Q. Cheng, and E. Ma, Full icosahedra dominate local order in $\mathrm{Cu}_{64} \mathrm{Zr}_{34}$ metallic glass and supercooled liquid, Acta Mater. 69, 343 (2014).
[81] J. Ding, Y.-Q. Cheng, and E. Ma, Charge-transfer-enhanced prism-type local order in amorphous $\mathrm{Mg}_{65} \mathrm{Cu}_{25} \mathrm{Y}_{10}$ : Short-tomedium-range structural evolution underlying liquid fragility and heat capacity, Acta Mater. 61, 3130 (2013).

[82] N. Wang, J. Ding, F. Yan, M. Asta, R. O. Ritchie, and L. Li, Spatial correlation of elastic heterogeneity tunes the deformation behavior of metallic glasses, npj Comput. Mater. 4, 19 (2018). 